\title{
THERMAL EXPANSION OF L-ASCORBIC ACID
}

B. Nicolai ${ }^{\mathrm{a}, *}$, M. Barrio ${ }^{\mathrm{b}}$, J.-Ll. Tamarit ${ }^{\mathrm{b}}$, R. Céolin ${ }^{\mathrm{c}}$, I.B. Rietveld ${ }^{\mathrm{a}}$

${ }^{a}$ Caracterisation des Matériaux Moléculaires à Activité Thérapeutique (CAMMAT), Faculté de Pharmacie, Université Paris Descartes, 75006 Paris, France

${ }^{\mathrm{b}}$ Grup de Caracteritzacio de Materials (GCM), Departament de Fisica i Enginyeria Nuclear, Universitat Politecnica de Catalunya, ETSEIB, 08028 Barcelona, Spain

cLETIAM, EA7357, IUT Orsay, université Paris Sud, rue Noetzlin, 91405 Orsay Cedex

* Corresponding author: beatrice.nicolai@parisdescartes.fr

\section{Abstract}

The specific volume of vitamin $\mathrm{C}$ has been investigated by $\mathrm{X}$-ray powder diffraction as a function of temperature from $110 \mathrm{~K}$ up to complete degradation around $440 \mathrm{~K}$. Its thermal expansion is relatively small in comparison with other organic compounds with an expansivity $\alpha_{\mathrm{v}}$ of $1.2(3) \times 10^{-4} \mathrm{~K}^{-1}$. The structure consists of strongly bound molecules in the $a c$ plane through a dense network of hydrogen bonds. The thermal expansion is anisotropic. Along the $b$ axis, the expansion has most leeway and is about 10 times larger than in the other directions. 


\section{Introduction}

As mentioned in the paper on the thermodynamic origin of phase diagrams by Céolin and Rietveld in this special issue [1], temperature - volume (T-v) phase diagrams are the projections of the intersections of the tangential lines connecting the Helmholtz free energy surfaces for the different phases of a system. Thus, the thermal expansion of a solid represents its T-v equilibrium with its vapor phase, which is part of the larger picture of the phase behavior of a chemical substance, which can be depicted as derivations of the Gibbs, Helmholtz, and internal energy and the enthalpy. Although it is not common to present phase behavior in the form of T-v phase diagrams, as pressure-temperature phase diagrams are more commonly used, certain systems do benefit from the T-v representation, in particular if their vapor pressure is significant, such as for the organic compound camphor [2] and the metalloid arsenic [3].

$\mathrm{L}$-ascorbic acid or vitamin $\mathrm{C}$ is known for its antiscorbutic and antioxidant properties. It is a labile water-soluble compound that degrades on heating and even in air at room temperature. In addition, it is known to degrade on storage in solution at room temperature. The influence of temperature on the chemical stability of L-ascorbic acid has mainly been studied in solution and thermal decomposition has rarely been studied in the solid state; only calorimetric and gravimetric data are available in the literature [4,5]. In addition, although the crystal structure of vitamin $\mathrm{C}$ is known for years [6], both from single-crystal X-ray and neutron diffraction measurements at and below room temperature [6-13], the influence of heating on crystalline Lascorbic acid has never been studied by X-ray diffraction.

A search in the Cambridge Structural Database (CSD) for crystal structures of L-ascorbic acid resulted in a number of structure files that all contained the same space group and unit cell. The references of the most useful CSD files have been compiled in Table 1 together with a few other publications on the structure [6-11]. L-ascorbic acid crystallizes in the monoclinic system with the non-centrosymmetric space group $P 2{ }_{1}$, with 4 molecules per unit-cell $(Z=4)$. In addition, several possible structures of L-ascorbic acid have been found by a number of different simulation approaches [14]. Some of those have an energy relatively close to the known structure [14], so it would be interesting to investigate by temperature dependent X-ray diffraction whether transient phases could be observed.

A possible degradation product of L-ascorbic acid through dimerization is dehydro-Lascorbic acid, which crystallizes in the monoclinic system as well, with space group $C 2$ and with 
two dimers in the unit cell $(Z=2)$. A preliminary report on its crystal structure was published by Hvoslef [12], who later published a complete description of the structure [13]. Literature data has been compiled in Table 1.

The thermal behavior of L-ascorbic acid, presented in this paper, will be used to investigate the presence of any transient structures on heating. Furthermore, the intermolecular interactions in the crystal of L-ascorbic acid will be investigated with the help of the thermal expansion tensor in combination with its structural characteristics such as hydrogen bonds.

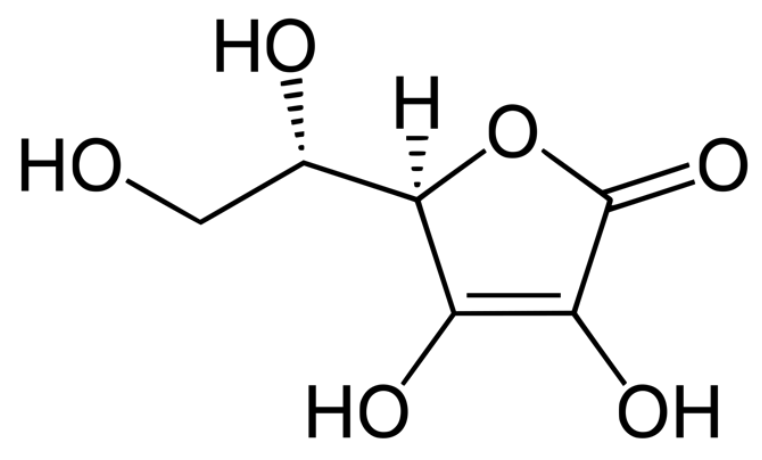

Figure 1. L-ascorbic acid or vitamin $\mathrm{C}, \mathrm{C}_{6} \mathrm{H}_{8} \mathrm{O}_{6},(R)$-3,4-dihydroxy-5-((S)-1,2 dihydroxyethyl)furan-2(5H)-one, $M=176.12 \mathrm{~g} \mathrm{~mol}^{-1}$

Table 1. Crystal data in the literature of L-ascorbic acid and dehydro-L-ascorbic acid

\begin{tabular}{|c|c|c|c|c|c|c|}
\hline $\mathbf{T} / \mathbf{K}$ & $a / \AA$ & $b / \AA$ & $c / \AA$ & $\beta /^{\circ}$ & $V / \AA^{3}$ & Ref / CSD ${ }^{a}$ \\
\hline \multicolumn{7}{|c|}{ L-ascorbic acid } \\
\hline $\mathrm{RT}^{\mathrm{b}}$ & 17.20 & 6.37 & 6.40 & $102^{\circ} 05^{\prime}$ & 685.67 & {$[6]$} \\
\hline $\mathrm{RT}^{\mathrm{b}}$ & $17.299(8)$ & $6.353(3)$ & $6.411(3)$ & $102.18(13)$ & 688.712 & {$[7,8] /$ LASCAC01, 10} \\
\hline 120 & $6.390(1)$ & $6.262(1)$ & $17.127(4)$ & $99.36(2)$ & 676.198 & [9] / LASCAC12 \\
\hline 100 & $6.395(2)$ & $6.240(2)$ & $17.095(3)$ & $99.341(9)$ & 673.129 & [10] / LASCAC13 \\
\hline 100 & $6.396(6)$ & $6.242(2)$ & $17.099(10)$ & $99.33(4)$ & 673.627 & [11] / LASCAC14 \\
\hline \multicolumn{7}{|c|}{ dehydro-L-ascorbic acid $\left(\mathrm{C}_{12} \mathrm{H}_{12} \mathrm{O}_{12}, \mathrm{M}=348.22 \mathrm{~g} \mathrm{~mol}^{-1}\right)$} \\
\hline $\mathrm{RT}^{\mathrm{b}}$ & 15.69 & 5.55 & 9.47 & 130.2 & 629.86 & {$[12]$} \\
\hline $\mathrm{RT}^{\mathrm{b}}$ & $15.728(9)$ & $5.530(2)$ & $9.453(5)$ & $130.56(4)$ & 624.64 & {$[13]$} \\
\hline
\end{tabular}

${ }^{\mathrm{a}}$ CSD: Cambridge Structural Database

${ }^{\mathrm{b}} \mathrm{RT}$ is room temperature $(283-303 \mathrm{~K})$ 


\section{Experimental}

\subsection{Vitamin C}

L-ascorbic acid of a purity $\geq 99 \%$ was purchased from Sigma-Aldrich. It is a white powder, which was used as such for the experiments after initial X-ray measurements demonstrated that its diffraction pattern was identical to the one calculated from the CIF file in the Cambridge Structural Database (CSD ref LASCAC10) with no extra peaks [8].

\subsection{High resolution X-ray powder diffraction}

A Debye-Scherrer geometry was used in combination with an INEL X-ray powder diffractometer equipped with a cylindrical position-sensitive detector (CPS120) containing 4096 channels $\left(0.029^{\circ} 2 \theta\right.$ angular step) and monochromatic $\mathrm{Cu} K \alpha_{1}(\lambda=1.54061 \AA)$ radiation.

For the measurements as a function of temperature, a liquid nitrogen 700 series Cryostream Cooler from Oxford Cryosystems (UK) was used. Slightly ground specimens were introduced in a Lindemann capillary ( $0.5 \mathrm{~mm}$ diameter) rotating perpendicularly to the $\mathrm{X}$-ray beam during the experiments to improve the average over the crystallite orientations. The temperature-dependent measurements were carried out from $110 \mathrm{~K}$ up to $440 \mathrm{~K}$, while the sample temperature was allowed to equilibrate for $10 \mathrm{~min}$ after changing the temperature to a new value. The heating rate in between data collection was $1.33 \mathrm{~K} \mathrm{~min}^{-1}$. The X-ray powder diffraction data were analyzed with the software FULLPROF using pattern matching [15].

\subsection{Isobaric thermal expansion tensor}

The anisotropy of the intermolecular interactions was investigated with the isobaric thermal expansion tensor, which is a measure of how the interactions change with temperature [16]. The tensor was calculated from the temperature-dependent lattice parameters with the program DEFORM [17]. Details of the procedure for the calculation of the tensor have been published elsewhere [16,18-21].

Decomposition of the tensor matrix yields the magnitude (eigenvalue) and orientation (eigenvector) of the thermal expansion along the 3 principal axes, $\mathbf{e}_{1}, \mathbf{e}_{2}$, and $\mathbf{e}_{3}$. The tensor indicates the directions of the least and most deformation as a function of temperature and these directions are commonly referred to as hard and soft directions, respectively, because they are related to the intermolecular interactions. The anisotropy of the thermal expansion of the intermolecular interactions can be condensed in the aspherism coefficient A [18,22,23]. 
Graphical representations of the tensor at given temperatures have been drawn using Wintensor [24].

\section{Results}

The diffraction patterns obtained as a function of temperature from 110 up to $440 \mathrm{~K}$ can be seen in Figure 2. Indexing of the patterns has led to the temperature dependence of the lattice parameters (See Table S1 in the Supplementary Information), which are linear except for parameter $c$, which has been fitted with a quadratic function.

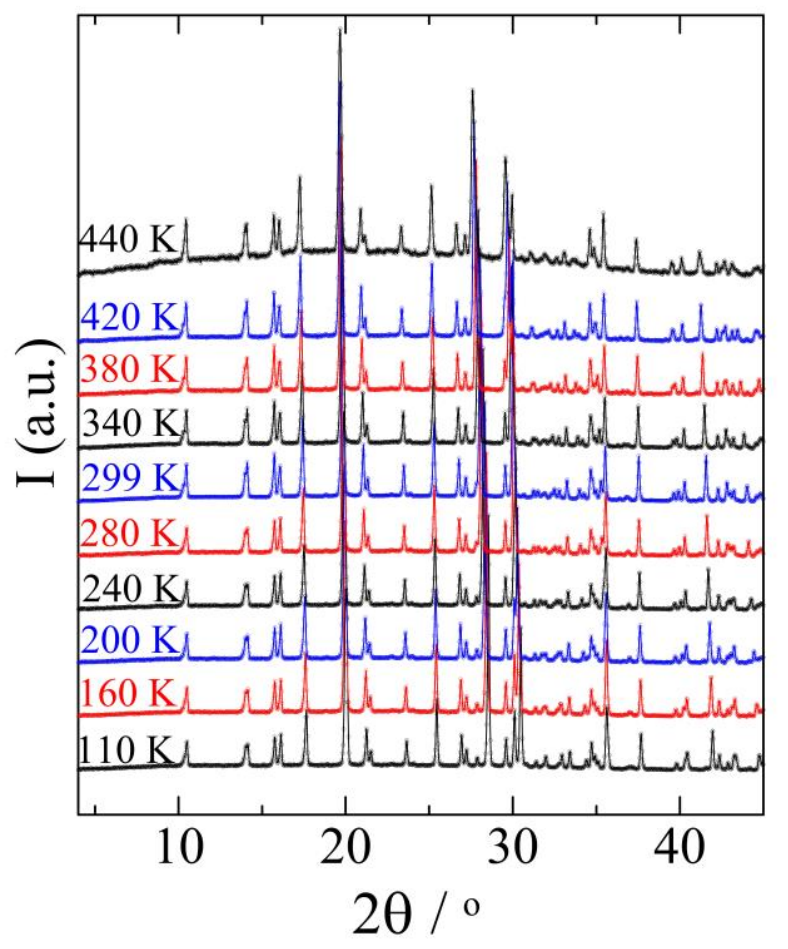

Figure 2. The diffraction patterns of vitamin C from $110 \mathrm{~K}$ up to $440 \mathrm{~K}$. At $440 \mathrm{~K}$, the sample partly degrades into a non-crystalline material, as indicated by the upward curvature of the baseline.

$$
\begin{array}{ll}
a / \AA=17.243(2)+1.69(8) \times 10^{-4} T / \mathrm{K} & \left(\mathrm{r}^{2}=0.983\right) \\
b / \AA=6.175(4)+6.09(12) \times 10^{-4} T / \mathrm{K} & \left(\mathrm{r}^{2}=0.997\right) \\
c / \AA=6.3929(12)+2.6(9) \times 10^{-5} \mathrm{~T} / \mathrm{K}+9.4(1.6) \times 10^{-8} \times T^{2} / \mathrm{K}^{2} & \left(\mathrm{r}^{2}=0.997\right)
\end{array}
$$


$\beta$, the only non- $90^{\circ}$ angle in the monoclinic cell, remains virtually constant with an average value of $102.08(4)^{\circ}$. The extremes are $102.125(1)$ at $110 \mathrm{~K}$ and $102.015(4)$ at $440 \mathrm{~K}$. The volume of the unit-cell, $V$, is very similar to the literature data and its change as a function of temperature was found to be linear as well:

$$
V / \AA^{3}=664.5(6)+0.0821(20) T / \mathrm{K} \quad\left(\mathrm{r}^{2}=0.995\right)
$$

With eq. 4, the expansivity of L-ascorbic acid, $\alpha_{\mathrm{V}}$, can be calculated, defined as $V=V_{0}\left(1+\alpha_{\mathrm{V}} T\right)$. $V_{0}=664.5(6) \AA^{3}$, which has been obtained from eq. 4 and this leads to a value for $\alpha_{\mathrm{V}}$ of $1.2(3) \times$ $10^{-4} \mathrm{~K}^{-1}$.

The unit-cell data has been used as input for the DEFORM program [17], which has led to the thermal expansion eigenvalues in combination with the aspherism coefficient $A$ [18]. The results have been reported in Table 2 . 
Table 2. Thermal expansion eigenvalues of monoclinic L-ascorbic acid as a function of temperature and aspherism coefficient $A$

\begin{tabular}{|c|c|c|c|c|}
\hline $\boldsymbol{T} / \mathbf{K}$ & $\boldsymbol{\alpha}_{11} \times \mathbf{1 0}^{-4}$ & $\boldsymbol{\alpha}_{\mathbf{2 2}} \times \mathbf{1 0}^{-\mathbf{4}}$ & $\boldsymbol{\alpha}_{33} \times \mathbf{1 0}^{-4}$ & $\boldsymbol{A}$ \\
\hline 110 & 0.975 & 0.147 & 0.0839 & 0.476 \\
\hline 160 & 0.970 & 0.147 & 0.0838 & 0.475 \\
\hline 200 & 0.967 & 0.147 & 0.0838 & 0.475 \\
\hline 240 & 0.963 & 0.147 & 0.0837 & 0.474 \\
\hline 280 & 0.959 & 0.147 & 0.0837 & 0.474 \\
\hline 299 & 0.957 & 0.147 & 0.0837 & 0.473 \\
\hline 340 & 0.954 & 0.147 & 0.0836 & 0.473 \\
\hline 380 & 0.950 & 0.147 & 0.0836 & 0.472 \\
\hline 420 & 0.946 & 0.147 & 0.0835 & 0.472 \\
\hline 440 & 0.943 & 0.147 & 0.0835 & 0.471 \\
\hline
\end{tabular}

\section{Discussion}

The diffraction patterns as a function of temperature in the range of $110 \mathrm{~K}$ to $460 \mathrm{~K}$ consistently remained that of the known structure of L-ascorbic acid; hence, no evidence for dimerization has been observed nor did the data point to any phase transformations into polymorphs predicted by computer-aided methods [14]. Only a decrease in the intensity of the Bragg peaks was observed, indicating that L-ascorbic acid slowly degrades, while at elevated temperatures.

All the unit-cell parameters increase with temperature, while the $\beta$ angle remains constant; hence, the unit-cell volume increases with the temperature, as expected. The eigenvalues of the thermal expansion tensor have been compiled in Table 2, while a graphical representation of the tensor at ambient temperature is presented in Figure 3. The expansivity $\alpha_{\mathrm{v}}$ equals $1.2(3) \times 10^{-4} \mathrm{~K}^{-}$ ${ }^{1}$, about half of the average value of $2 \times 10^{-4} \mathrm{~K}^{-1}$ for small organic molecules [25-27].

The aspherism coefficient has a value of 0.47 , very similar to that of tienoxolol [21], indicating that the thermal expansion is anisotropic over the entire observed temperature range 
(Table 2). The expansion in the crystal structure mainly occurs along the $b$ axis, which coincides with the tensor axis $\mathbf{e}_{1}$ and has the eigenvalue $\alpha_{11}$ of 0.957 at $299 \mathrm{~K}$, which is between 6 to 10 times larger than the expansion along the two other axes. The high value implies that the $b$ axis, or $\mathbf{e}_{1}$, is the, relatively speaking, soft direction of the crystal.

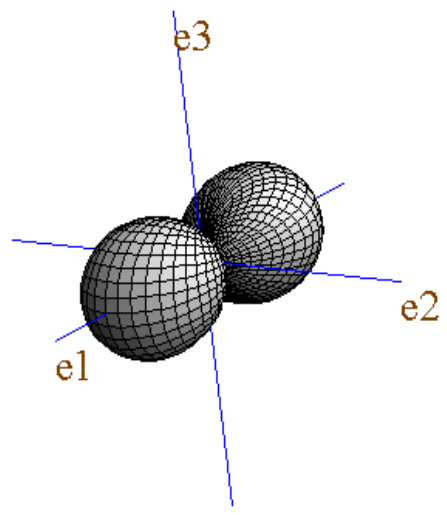

Figure 3. Graphical representation of the tensor at room temperature (drawn by Wintensor [24]). The expansion at room temperature is very limited in the $\mathbf{e}_{\mathbf{2}}$ and $\mathbf{e}_{\mathbf{3}}$ direction and large in the $\mathbf{e}_{\mathbf{1}}$ direction.

The furanone $\left(\mathrm{C}_{4} \mathrm{O}_{2}\right)$ rings of the two molecules in the asymmetric unit are both parallel to the ac plane. The two molecules (A and B) differ in the conformation of their alkyl chain. Each molecule is involved in 10 hydrogen bonds with at least 6 different molecules. Each molecule acts as a hydrogen bond donor and an acceptor. The hydrogen bonds are all of type $\mathrm{O}-\mathrm{H}^{\cdots} \mathrm{O}$ and all the hydroxyl groups are involved in bifurcated hydrogen bonds. There are 3 infinite hydrogen bond chains with graph-set notation $C^{1}{ }_{1}(6), C^{1}{ }_{1}(7)$, and $C^{1}{ }_{1}(7)$ [28,29]. One infinite chain is perpendicular to the $c$ axis made up of $\mathrm{O}-\mathrm{H}^{\cdots} \mathrm{O}_{\text {hydroxyl }}$ bonds and is formed alternatingly by the two molecules (A and B) of the asymmetric unit (see Figure S1 in the Supplementary Information). One infinite chain is parallel to the $c$ axis. It involves bifurcated hydrogen bonds of the type $\mathrm{O}-\mathrm{H}^{\cdots} \mathrm{O}_{\text {ring, }}$, and contains only A-type molecules and a second chain only B-type molecules (See Figure S2 in supplementary Information). Finally, there is a zigzag hydrogen bonds parallel to the $b$ axis. It involves bifurcated hydrogen bonds between A and B molecules as 
well (See Figure S3 in supplementary Information). All the hydrogen bonds are more or less perpendicular to the $\mathrm{b}$ axis and they form therefore sheets of molecules that are internally strongly bound. The maximum expansion occurs, therefore, perpendicular to the sheets along the $b$ axis as illustrated in Figure 4.

The expansion in the $a c$ plane along $\mathbf{e}_{\mathbf{2}}$ is almost twice as large as the expansion along $\mathbf{e}_{3}$, as can be seen in Table 2 and Figures 3 and 4. $\mathbf{e}_{2}$ runs more or less along the $c$ axis (Table S1 in the Supplementary Information). It appears therefore that the hydrogen bond network along the $a$ axis has somewhat more leeway to move than the network in the direction of the $c$ axis. It must be said however, that even along the $b$ axis the sheets are kept together by hydrogen bonds. Due to the positioning of these hydrogen bonds mainly within the $a c$ plane, the latitude for displacement along the $b$ axis is rather large in comparison to the two other directions.
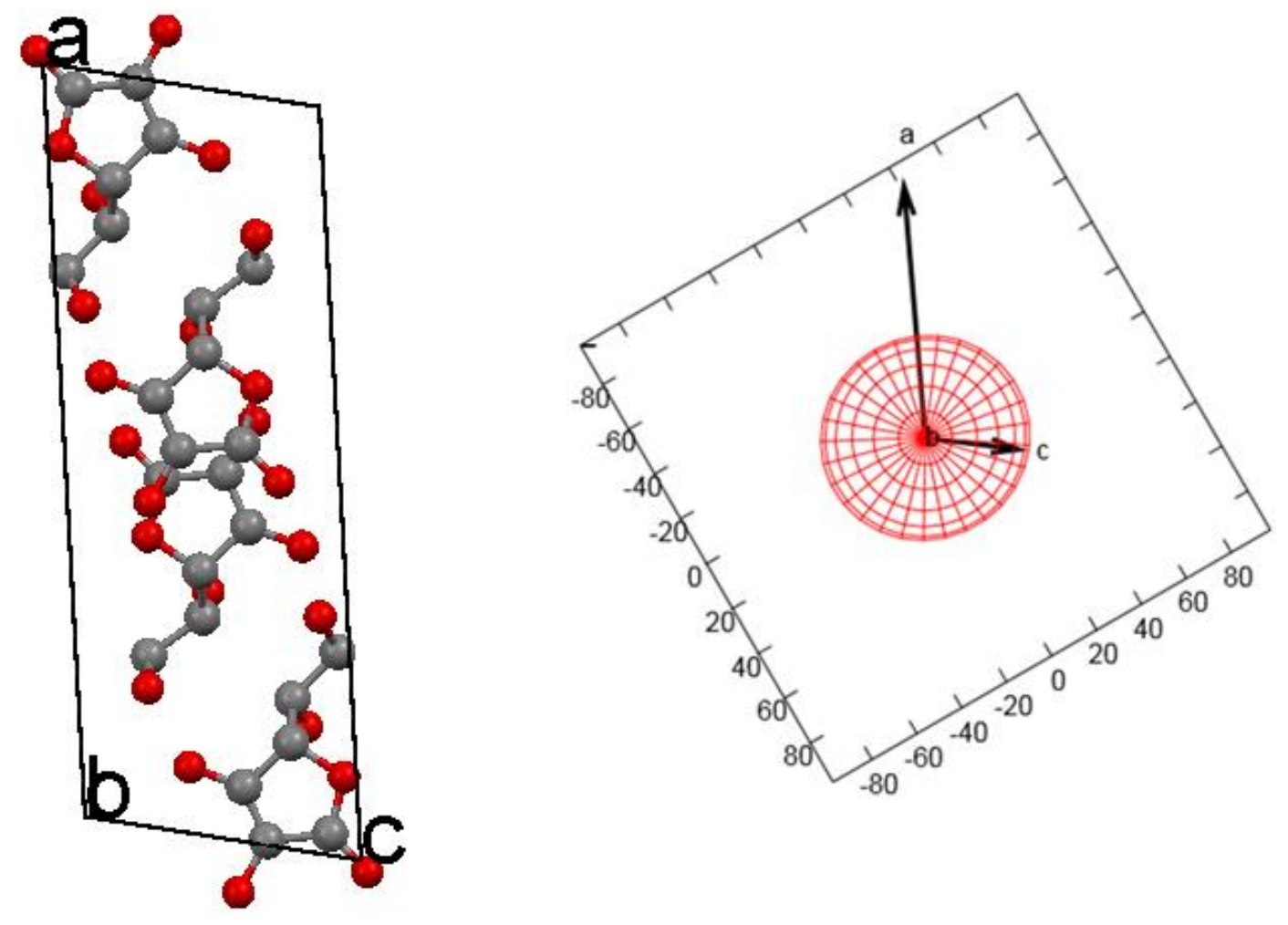

a 

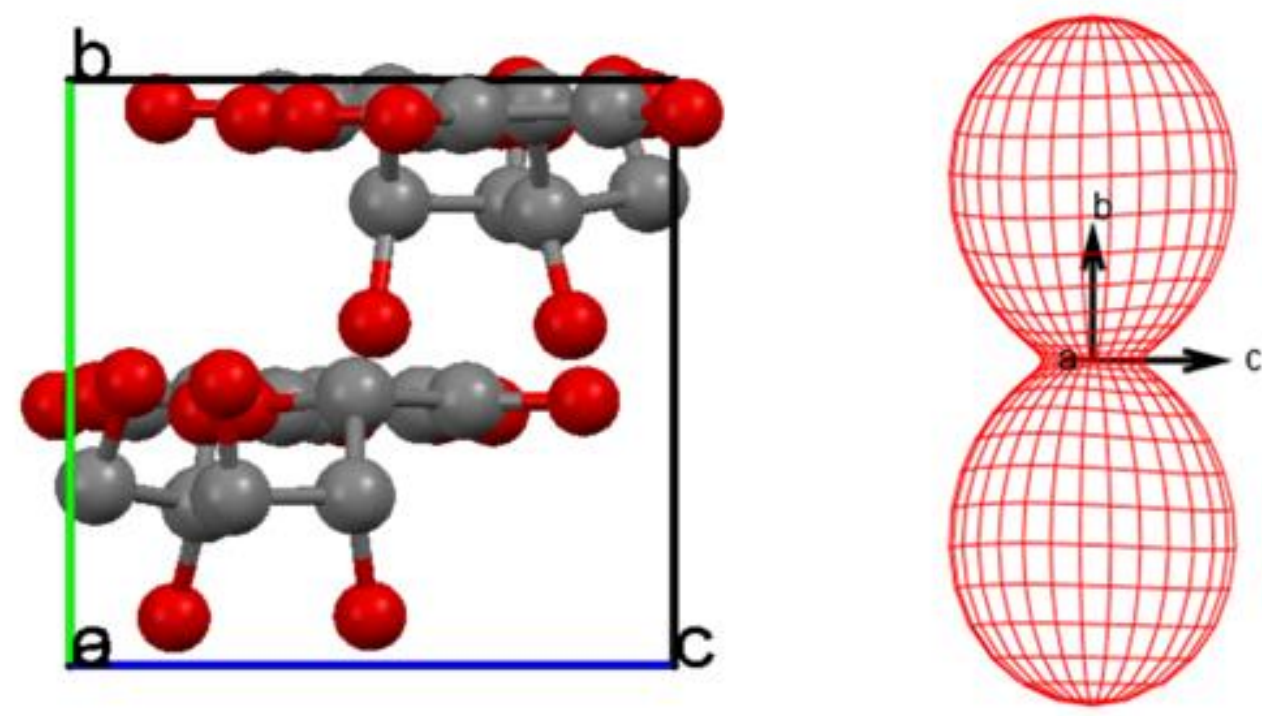

b
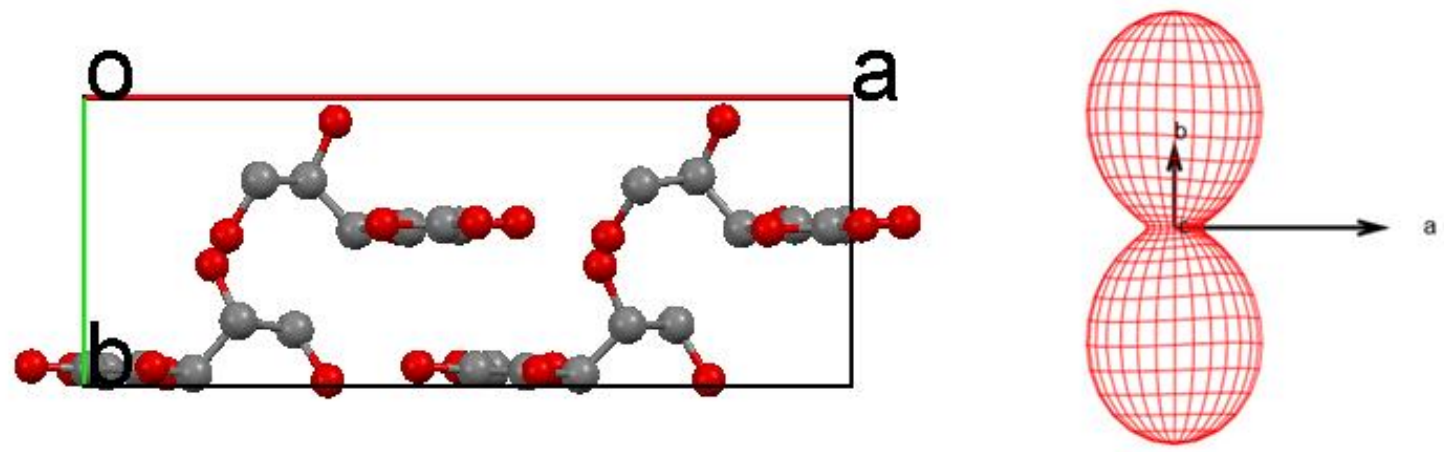

$\mathrm{C}$

Figure 4. Projection of the tensor in 3 different crystallographic planes (a) perpendicular to the $b$ axis revealing the molecular sheets with tightly bound molecules through hydrogen bonds, (b) perpendicular to the $a$ axis revealing the layers of molecules that have most leeway to expand, and (c) perpendicular to the $c$ axis. (drawn by PASCAL [28]). The orientation of the principal axes of the tensor $\left(\mathbf{e}_{1}, \mathbf{e}_{2}\right.$, and $\left.\mathbf{e}_{3}\right)$ in the crystal is given in table $\mathrm{S} 2$ of the Supplementary Information. 


\section{Conclusion}

Up to a temperature of $440 \mathrm{~K}$, no phase transition or dimerization of crystalline L-ascorbic acid has been observed with X-ray powder diffraction. With an expansivity of $\alpha_{\mathrm{V}}=1.2(3) \times 10^{-4}$ $\mathrm{K}^{-1}$, the thermal expansion is fairly small in comparison with other organic molecules. Moreover, the thermal expansion is anisotropic over the entire temperature range with the $b$ axis of the unit cell exhibiting most expansion. The molecules are held together by a large number of hydrogen bonds, which form a strong network in the ac plane. This limits the expansion in this plane, resulting in the larger expansion along the $b$ axis, where the awkward positioning of the hydrogen bonds relative to the $b$ axis give this direction more leeway for expansion.

\section{Acknowledgement}

This work has been supported by the Spanish Ministry of Science and Innovation (grants FIS2014-54734-P) and the Catalan Government (grant 2014 SGR-581).

\section{References}

1. R. Ceolin and I. B. Rietveld, Eur. Phys. J. - S.T. (2017).

2. I. B. Rietveld, M. Barrio, N. Veglio, P. Espeau, J.-L. Tamarit, and R. Céolin, Thermochim. Acta 511 (1-2), 43 (2010).

3. J.-C. Rouland, R. Ceolin, C. Souleau, and P. Khodadad, J. Therm. Anal. Calorim. 23 (12), 143 (1982).

4. G. Vernin, S. Chakiba, S. M. Rogachevab, T. D. Obretenov, and C. Pakanyic, Carbohydr. Res. 305 (1), 1 (1997).

5. M. Juhasz, Y. Kitahara, S. Takahashi, and T. Fujii, J. Pharm. Biomed. Anal. 59, 190 (2012).

6. J. Hvoslef, Acta Chem. Scand. 18 (1), 841 (1964).

7. J. Hvoslef, Acta Crystallogr. B 24, 1431 (1968).

8. J. Hvoslef, Acta Crystallogr. B 24, 23 (1968).

9. M. Milanesio, R. Bianchi, P. Ugliengo, C. Roetti, and D. Viterbo, J. Mol. Struct.: THEOCHEM 419, 139 (1997).

10. B. Dittrich, M. Strumpel, M. Schafer, M. A. Spackman, and T. Koritsanszky, Acta Crystallogr. A 62, 217 (2006).

11. I. A. Guzei (CSD LASCAC14 - L-ascorbic acid structure).

12. J. Hvoslef, Acta Chem. Scand. 24, 2238 (1970).

13. J. Hvoslef, Acta Crystallogr. B 28, 916 (1972).

14. A. Arslantas, W. C. Ermler, R. Yazici, and D. M. Kalyon, Int. J. Mol. Sci. 6, 291 (2005).

15. J. Rodriguez-Carvajal, Physica B 192, 55 (1993).

16. J. Salud, M. Barrio, D. O. Lopez, J. L. Tamarit, and X. Alcobe, J. Appl. Crystallogr. 31, 748 (1998). 
17. A. Filhol, J. Lajzerowicz, and M. Thomas, DEFORM (1987).

18. P. Negrier, L. C. Pardo, J. Salud, J. L. Tamarit, M. Barrio, D. O. Lopez, A. Wurflinger, and D. Mondieig, Chem. Mater. 14 (5), 1921 (2002).

19. B. Nicolaï, N. Mahé, R. Céolin, I. B. Rietveld, M. Barrio, and J.-L. Tamarit, Struct. Chem. 22 (3), 649 (2011).

20. A. D. Fortes, I. G. Wood, and K. S. Knight, Phys. Chem. Miner. 35 (4), 207 (2008).

21. B. Nicolaï, I. B. Rietveld, M. Barrio, N. Mahé, J.-L. Tamarit, R. Céolin, C. Guéchot, and J.-M. Teulon, Struct. Chem. 24 (1), 279 (2013).

22. D. Weigel, T. Beguemsi, P. Garnier, and J. F. Berar, J. Solid State Chem. 23 (3-4), 241 (1978).

23. P. Negrier, M. Barrio, J. L. Tamarit, N. Veglio, and D. Mondieig, Cryst Growth Des 10 (6), 2793 (2010).

24. W. Kaminski, WinTensor (http://www.wintensor.com) (2004).

25. R. Céolin and I. B. Rietveld, Ann. Pharm. Fr. 73 (1), 22 (2015).

26. I. B. Rietveld and R. Céolin, J. Therm. Anal. Calorim. 120 (2), 1079 (2015).

27. A. Gavezzotti, Molecular Aggregation. Structure Analysis and Molecular Simulation of Crystals and Liquids. (Oxford University Press, Oxford, UK, 2013).

28. M. C. Etter, Acc. Chem. Res. 23 (4), 120 (1990).

29. M. C. Etter, J. C. Macdonald, and J. Bernstein, Acta Crystallogr. B 46, 256 (1990).

28. M. J. Cliffe and A. L. Goodwin, J. Appl. Crystallogr. 45, 1321 (2012). 